... Elisabeth Müller: Allgemeinmedizinerin, Gründerin des «Kafi Mümpfeli»

in Zürich und «Kopf des Jahres» des Kollegiums für Hausarztmedizin

\title{
«In der Sprechstunde ist man vom Sprechen weggekommen»
}

Daniel Lüthi

Text und Bilder
Das Kafi Mümpfeli* ist ein modern eingerichtetes Restaurant mit einer vielversprechenden Speisekarte, einer schönen Terrasse und rund 50 Plätzen. Es liegt nur wenige hundert Meter von der Hausarztpraxis von Elisabeth Müller entfernt - und ist eigentlich eine Art «Verlängerung», eine Filiale dieser Praxis. Denn von den insgesamt 22 Angestellten des Restaurants, das sich etwas gar bescheiden «Kafi» nennt, sind fast alle Patientinnen und Patienten von Müller. Sie selber steht übrigens mindestens einen Tag pro Woche als Köchin in der Küche, «allein über Mittag gehen bis zu 60 Essen raus, dann kommt noch das
Abwaschen und Putzen, das gehört selbstverständlich auch bei mir dazu. Hier bin ich nicht die Frau Doktor, sondern eine gleichwertige Mitarbeiterin, die erledigt, was ansteht.» Ein bescheidener Mensch, diese Ärztin, das wird sofort klar. Eine Frau auch, die Sachen macht, statt bloss darüber zu reden.

Übrigens: Das Kafi Mümpfeli ist an 365 Tagen im Jahr geöffnet.

\section{Erzählen und zuhören können}

Ein Café fürs Quartier, ein Ort, wo man sich treffen kann und sitzenbleiben, solange man will, ein Res-

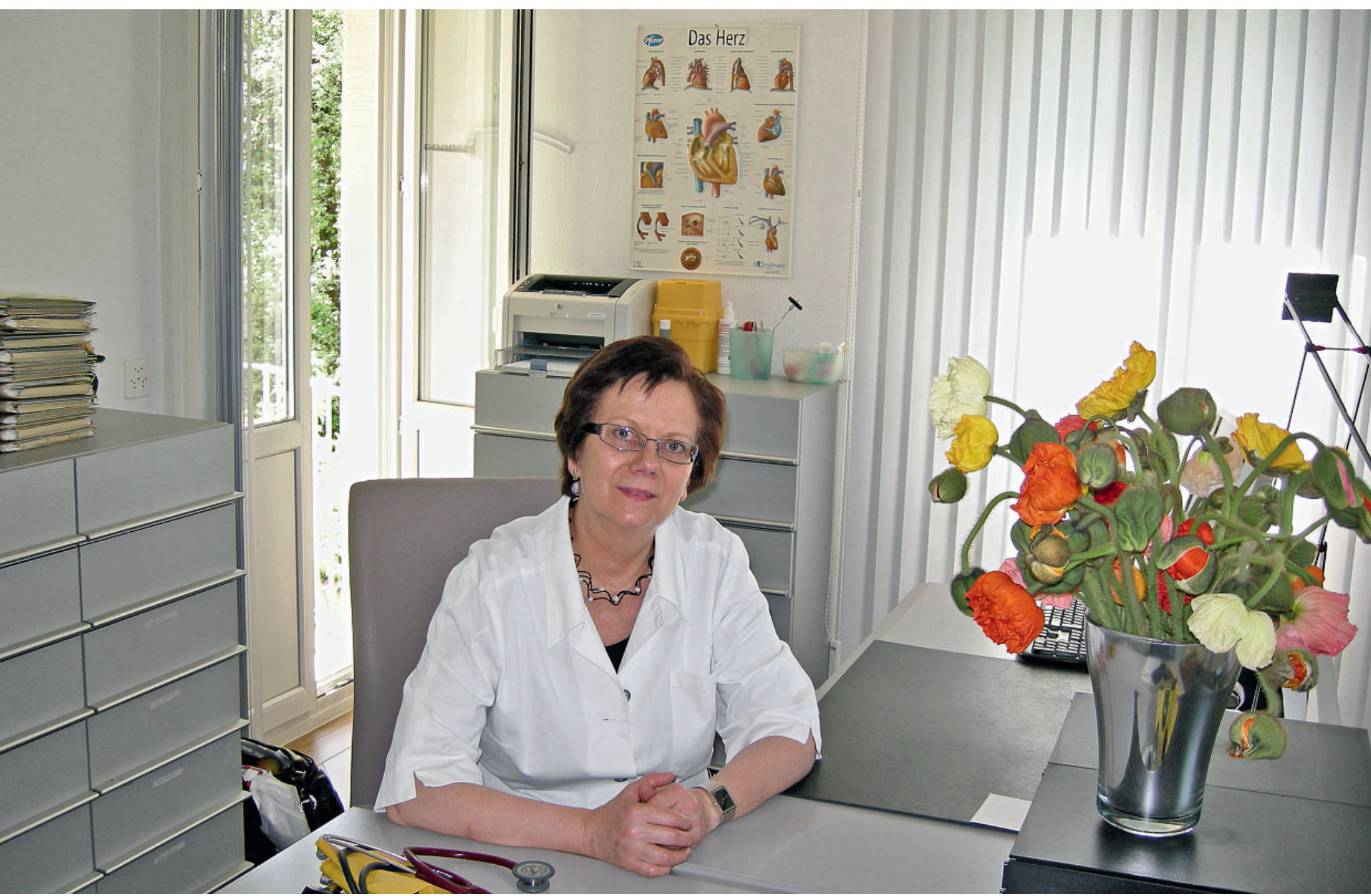


taurant mit sozialem Hintergrund und günstigen Preisen: Das war schon lange die Idee von Elisabeth Müller. Im Juni 2009 setzte sie den Traum in die Wirklichkeit um, mit eigenem Geld und viel Engagement, «aber ohne Business- oder Budgetplan. Ich weiss, dass es blauäugig, ja unprofessionell war, wie ich an dieses Projekt heranging. Ich weiss aber auch, dass man manchmal unprofessionell handeln muss, weil man sonst nämlich zu viel überlegt und plant - und nichts macht.»

Eine Ärztin, die ein Restaurant eröffnet und dieses parallel zu ihrer Praxis führt - das ist ungewöhnlich, für Elisabeth Müller aber absolut naheliegend: «Druck und Hektik nehmen zu in der Arbeitswelt - gleichzeitig gibt es immer mehr Menschen, die sich einsam fühlen. Das ist etwas, was unsere Gesellschaft krank macht: dass wir uns gegenseitig nicht mehr zuhören können und wollen, auch in der Familie nicht. Leider ist es auch bei vielen Ärztinnen und Ärzten so: In der Sprechstunde ist man vom Sprechen weggekommen. Patienten erzählen mir von Spezialisten, die ihnen den Rücken zeigen und ins Diktaphon sprechen. Von einem Arzt, der den Patienten anschnauzte: 〈Hier stelle ich die Fragen, nicht Sie.> Oder einem, der unvermittelt aufstand, dem Patienten die Hand entgegenstreckte und sich verabschiedete - als dieser noch etwas sagen oder fragen wollte. Auch habe ich von Gynäkologen gehört, bei denen die Frauen schon ausgezogen daliegen müssen, wenn der Herr Doktor kommt.»

\section{Offene Ohren}

Zu Elisabeth Müller kommen, so erzählt sie, viele psychisch Angeschlagene. Weil es sich schnell herumspricht, dass man hier noch sprechen kann und einem jemand zuhört. «Aber auch psychisch Gesunde erzählen manchmal gerne etwas und sagen dann am Schluss der Sprechstunde, jetzt gehe es ihnen schon viel besser. Ohne Spritze, und ohne Rezept.» Ein offenes Ohr als Therapieform. Oder wie sie selber sagt: «Ich versuche immer, mich in mein Vis-à-vis hineinzuversetzen.» Empathie als therapeutischer Grundsatz. Aber Elisabeth Müller ist, wie gesagt, ein bescheidener Mensch. Einer, der grosse Worte scheut und lieber im Kleinen etwas bewegt.

Das war einer der Beweggründe, die zur Gründung des Kafi Mümpfeli führten: die Erkenntnis, dass die Zeit in der Hausarztpraxis trotz allem nicht reichte, um allen, die es nötig gehabt hätten, genug zuhören zu können. «Im Gespräch mit meinen Patienten wurde mir klar, dass es neben der Praxis noch einen anderen Ort bräuchte, wo man reden kann. Ein Café oder Restaurant ist ein solcher Ort. Die sgruusigste Beiz ist eine wertvolle soziale Institution, wenn sie liebevoll geführt wird», sagt Elisabeth Müller.

\section{Behindert und doch normal}

Ihr zweiter Katalysator, selber eine Beiz zu eröffnen, war der: «Ich sehe immer wieder Leute, die durch alle

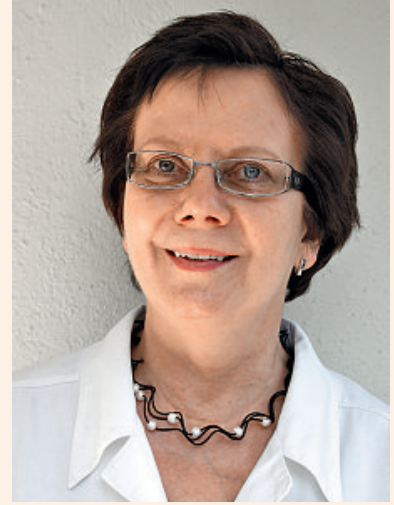

\section{Elisabeth Müller}

Dr. med. Elisabeth Müller wurde 1952 in Zürich geboren, wo sie auch die Schulen absolvierte. Sie liess sich zuerst zur Laborantin ausbilden und danach zur Krankenschwester. Zehn Jahre lang arbeitete sie am Kantonsspital Winterthur, machte dann auf dem zweiten Bildungsweg die Matura und studierte in Zürich Medizin. 1992 konnte sie in Zürich-Affoltern - etwas überraschend - die Hausarztpraxis eines Kollegen übernehmen, den sie zuvor mehrmals vertreten hatte. 2009 übernahm Elisabeth Müller ganz in der Nähe ihrer Praxis ein Restaurant und machte daraus ihr Projekt «Kafi Mümpfeli». Leute, die zwischen die sozialen Maschen fallen viele davon eigene Patientinnen und Patienten - finden dort Arbeit und Strukturen. Vor wenigen Tagen erhielt Elisabeth Müller für ihr Engagement vom Kollegium für Hausarztmedizin KHM im KKL Luzern die Auszeichnung «Kopf des Jahres».

Sie lebt mit ihrem Ehemann in Rapperswil am Zürichsee.

Maschen des sozialen Netzes fallen, Menschen, für deren Leiden niemand bezahlen will. Und solche, die krankgeschrieben und von IV und Sozialamt abgeschrieben sind, obschon sie arbeiten könnten - wollte man ihrer Behinderung im Arbeitsmarkt nur Rechnung tragen.» Müller nennt als Beispiel eine Frau, die Probleme mit beiden Schultern hat. Niemand wollte sie anstellen, ihren Mann, einen Handwerker, wagte sie kaum mehr um Geld für ein Kleidungsstück zu bitten, Beziehungsprobleme waren die Folge. Jetzt arbeitet sie, gemäss ihren Möglichkeiten - und deshalb glücklich im Kafi Mümpfeli. Man musste in der Küche nur das 
Material ein wenig tiefer lagern, damit sie jeweils ihre Arme nicht so hoch hinaufheben muss.

Im Kafi Mümpfeli arbeiten auch Leute, denen ihre Behinderung anzusehen ist. Sie verdienen gleich viel wie alle anderen. Und fühlen sich allein deshalb als ganz normale Arbeitnehmerinnen. «Es gibt aber auch Randgruppen, die im Alltag niemand als solche erkennen würde», weiss Elisabeth Müller. Auch sie gilt es ernst zu nehmen und am richtigen Ort einzusetzen. So dass der Gast letztlich kaum merkt, dass dieses Kafi eigentlich gar kein normales Restaurant ist.

\section{«Da schickte mir doch tatsächlich eine Krankenkasse einen Brief, in dem sie mich fragte, warum ich eine Salbe verschrieben habe, die 16 Franken 50 kostet.»}

\begin{abstract}
Ländliche Stadt
Zurück in die Arztpraxis: An der Wand hinter dem Pult hängt ein Schaubild mit dem Titel «Das Herz». Das Bild passt. Die Ärztin, die hier arbeitet, hat offensichtlich ein grosses Herz. Und viel Bodenhaftung. Das wiederum könnte mit ihrer Herkunft zu tun haben: Ihr Vater war Bergbauer in Brienz im Berner Oberland, die Mutter Handarbeitslehrerin aus Zürich. Sie wollte Tochter Elisabeth in ihrer Heimat zur Welt bringen, dort blieb diese dann auch. Während der letzten zwei Studienjahre und während der Zeit als Assistenzärztin vertrat Müller mehrmals einen Allgemeinmediziner. Als dieser völlig unerwartet verstarb, boten ihr seine Angehörigen die Praxis zum Kauf an. «Damals hatte man noch das Gefühl, ein solches Angebot annehmen zu müssen», sagt sie heute dazu. Den Schritt bereut sie nicht.

Elisabeth Müller gefällt der Standort ihrer Praxis in Zürich-Affoltern: «Es ist ein Ort mit ländlichem Charakter. Hier sind die Leute noch normal, nicht so überdreht wie anderswo, sie respektieren mich als Fachfrau und knallen mir nicht einfach einen Internet-Ausdruck auf den Tisch, um ihre Forderungen zu unterstreichen. Und im Wartezimmer ist immer ein grosses Hallo, weil sich die Leute gegenseitig ja kennen.» Elisabeth Müller liebt auch ihren Beruf: «Es ist faszinierend, ganze Familien und deren häusliche Verhältnisse kennenzulernen, manchmal über mehrere Generationen hinweg, und Beziehungen aufzubauen. Dies hilft mir, Situationen richtig einzuschätzen.»
\end{abstract}

\section{Zukunft mit Fragezeichen}

Etwas macht Müller gar nicht gern, nämlich den zunehmenden Bürokram erledigen: «Die Verbürokratisierung der Medizin ist eine Katastrophe», ärgert sich jetzt jemand, der sonst sehr ausgeglichen wirkt. «Da schickte mir doch tatsächlich eine Krankenkasse einen Brief, in dem sie mich fragte, warum ich eine Salbe verschrieben habe, die 16 Franken 50 kostet. Ich schrieb zurück, dass dieser Briefwechsel ein Vielfaches koste und allein deshalb unsinnig sei. Das ist doch nichts anderes als das Zeichen einer ungehörigen Bevormundung und eines aufgeblähten Apparates mit Leuten, die von Medizin keine Ahnung haben.»

Nicht zuletzt aus solchen Gründen würden immer weniger Studienabgänger die Allgemeinmedizin als ihr Spezialfach wählen. «Dazu kommt, dass wir im Vergleich mit den Spezialisten und dem, was wir leisten, zu wenig verdienen.» Immer wieder vom Essen wegrennen zu müssen oder in der Nacht einen Hausbesuch zu machen, sei eben auch nicht jedermanns Sache. «Viele Junge denken: «Warum sollte ich zwölf Stunden pro Tag schuften, wenn ich auch acht Stunden arbeiten kann?> Als Hausarzt kann man sich die Zeit oft halt nicht so einteilen, wie man gerne möchte.»

Fazit: Elisabeth Müller befürchtet, dass der Hausarztberuf dereinst aussterben könnte, «was ich sehr bedauern würde.» Und viele Patientinnen und Patienten sicher auch.

Dass das Kafi Mümpfeli nicht stirbt, ist auch immer noch ein Kampf. Seit rund einem halben Jahr ist die Trägerschaft eine Stiftung. Nach wie vor aber stopft Müller die finanziellen Löcher mit ihren eigenen Mitteln, es geht um mehrere tausend Franken pro Monat. «Unser Ziel ist es, dass diese Institution selbsttragend wird, das wäre das Allerschönste.» Die Idee von Patenschaften liegt in der Luft - Entwicklungshilfe vor der Haustüre wäre das. Sponsoring und Fundraising sind angesagt.

Und wer weiss: Vielleicht hilft ja auch die Auszeichnung weiter, die Elisabeth Müller Mitte Monat vom Kollegium für Hausarztmedizin erhalten hat der Titel «KHM Kopf des Jahres». «Den Schwachen in unserer Gesellschaft eine starke Hand reichen»-, für sie seien dies nicht bloss leere Worte, heisst es in der Laudatio.

Auf die Ehrung angesprochen sagt Müller selber: «Ich habe keinen blassen Schimmer, wie es dazu gekommen ist.» 